\title{
PMN-PT single crystal and Terfenol-D alloy magnetoelectric laminated composites for electromagnetic device applications
}

\author{
Yaojin WANG*,**, Kwok Fung CHEUNG*, Siu Wing OR ${ }^{*, \dagger}$, Helen Lai Wa CHAN*and Haosu LUo** \\ * Department of Applied Physics, The Hong Kong Polytechnic University, Hung Hom, Kowloon, Hong Kong \\ ${ }^{* *}$ State Key Laboratory of High Performance Ceramics and Superfine Microstructure, Shanghai Institute of Ceramics, \\ Chinese Academy of Sciences, Shanghai 201800, China
}

\begin{abstract}
Magnetoelectric laminated composites of a length-magnetized magnetostrictive $T_{b_{0.3}} \mathrm{Dy}_{0.7} \mathrm{Fe}_{1.92}$ (Terfenol-D) alloy plate sandwiched between two thickness-polarized, electro-parallel-connected piezoelectric $0.7 \mathrm{~Pb}\left(\mathrm{Mg}_{1 / 3} \mathrm{Nb}_{2 / 3}\right) \mathrm{O}_{3}-0.3 \mathrm{PbTiO} 3(\mathrm{PMN}-\mathrm{PT})$ single crystal plates were fabricated and their converse magnetoelectric properties were evaluated. An electromagnetic control device using a laminated composite as the control element and an Alnico biasing magnet, two Mn-Zn F-yokes and a Mu-metal movable plate as the magnetic circuit was developed and characterized. The distribution of the dc magnetic flux lines and magnetic inductions of the device were analyzed using a finite element method. The quasistatic and dynamic magnetic inductions of the device were measured, together with the generative forces. The results revealed that the device has linear voltagecontrolled magnetic inductions in excess of $0.6 \mathrm{G} / \mathrm{V}$ with flat responses in the measured frequency range of $0.1-40 \mathrm{kHz}$ and large generative forces over $0.7 \mathrm{~N}$ at $160 \mathrm{~V}$ at the designated gaps of 1.0-1.2 mm. This coil-free device could be useful in broadband and precision magnetic flux/force control applications with negligible Joule heating losses.
\end{abstract}

(C)2008 The Ceramic Society of Japan. All rights reserved.

Key-words: Converse magnetoelectric effect, Electromagnetic control device, Magnetoelectric laminated composite, Magnetostrictive alloy, Piezoelectric single crystal

[Received November 16, 2007; Accepted February 21, 2008]

1. Introduction

The direct magnetoelectric (DME) effect is an electric polarization response of a magnetoelectric (ME) material to an applied magnetic field, while the converse magnetoelectric (CME) effect is a magnetization response of the material to an applied electric field. ${ }^{1)}$ In the past decades, considerable research efforts have been put on the DME effect, first in single-phase materials, then in two-phase bulk composites, and lately in two/three-phase laminated composites. ${ }^{2)-6)}$ To date, it is known that laminated composites of piezoelectric $0.7 \mathrm{~Pb}\left(\mathrm{Mg}_{1 / 3} \mathrm{Nb}_{2 / 3}\right) \mathrm{O}_{3-}$ $0.3 \mathrm{PbTiO}_{3}$ (PMN-PT) single crystal and magnetostrictive $\mathrm{Tb}_{0.3} \mathrm{Dy}_{0.7} \mathrm{Fe}_{1.92}$ (Terfenol-D) alloy possess superior DME effect due to their greater product effect of the direct piezoelectric effect and the direct magnetostrictive effect. ${ }^{4)}$ In particular, their ultrahigh DME voltage coefficients $\left(\alpha_{\mathrm{V}} \sim 0.11 \mathrm{~V} / \mathrm{Oe}\right)$, defined by an induced electrical voltage in response to an applied ac magnetic field $(\mathrm{d} V / \mathrm{d} H)$, have led to the commercial development of power-free magnetic field sensing devices. ${ }^{7)}$

By contrast, few of information has been available for the CME effect in ME materials in general and in the laminated composites in specific. ${ }^{8,9)}$ In fact, it is both physically interesting and technologically important to quantitatively characterize the CME effect in terms of the CME flux coefficient $\left(\alpha_{\mathrm{B}}\right)$ defined by a magnetic induction in response to an applied ac voltage $(\mathrm{d} B /$ $\mathrm{d} V$ ). From the perspective of applications, ME materials with high $\alpha_{\mathrm{B}}$ are crucial to the direct realization of core-free electromagnetic control devices. Since the traditional devices for magnetic flux/force control are essentially constructed from coilwound electromagnets and operated according to Faraday's law

Corresponding author: Siu Wing OR; E-mail: apswor@polyu.edu. hk of electromagnetic induction, they suffer intrinsically from Joule heating losses, bandwidth limitations and precision problems. ${ }^{10)}$ Electromagnetic control devices based on the CME effect in $\mathrm{ME}$ materials do not need any magnetic coil, and thus have reduced Joule heating losses, improved operating bandwidths and increased control preciseness.

In this paper, we report a prominent CME effect in laminated composites consisting of one Terfenol-D alloy plate bonded between two electro-parallel-connected PMN-PT single crystal plates in the thickness direction, and present the development and performance of a coil-less electromagnetic control device formed by such a laminated composite, an Alnico biasing magnet, two Mn-Zn F-yokes and a Mu-metal movable plate.

2. PMN-PT and Terfenol-D magnetoelectric laminated composites

2.1 Structure and working principle

Figure 1 shows the schematic diagram and photograph of the proposed ME laminated composites with the CME effect. The composite has one layer of magnetostrictive Terfenol-D alloy sandwiched between two layers of piezoelectric PMN-PT single crystal arranged in reverse polarization (P) directions and connected electrically in parallel to form the input terminals. The PMN-PT plates were cut from a PMN-PT ingot grown in-house using a modified Bridgman technique. ${ }^{11)}$ The dimensions of the plates were $12 \mathrm{~mm}$ long, $6 \mathrm{~mm}$ wide and $1 \mathrm{~mm}$ thick. Their $<001>$ and $<011>$ crystallographic axes were arranged along the length and thickness directions, respectively. Polarization was induced using the $<011>$ axis in the thickness direction so as to impart a high transverse piezoelectric response to the plates with deformations along the length direction when excited electrically in the thickness direction. The piezoelectric coefficient $\left(d_{31, \mathrm{P}}\right)$ and 


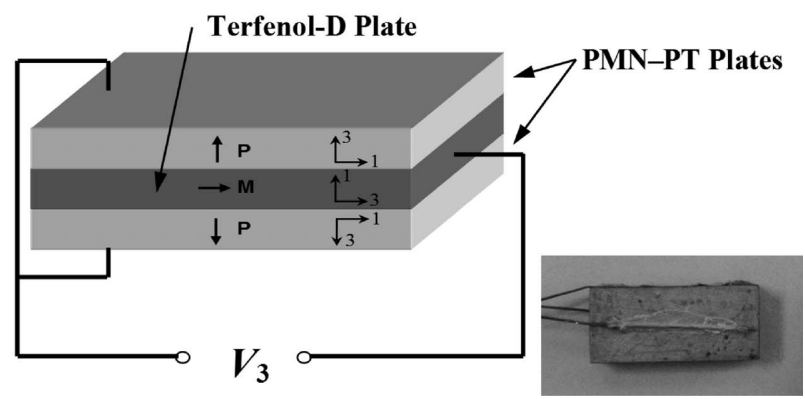

Fig. 1. Schematic diagram and photograph (the inset) of the proposed ME laminated composites with the CME effect.

elastic compliance coefficient at constant electric field $\left(s_{11}^{E}\right)$ of the PMN-PT plates were measured to be $-1630 \mathrm{pC} / \mathrm{N}$ and 125 $\mathrm{pm}^{2} / \mathrm{N}$, respectively. The Terfenol-D plate was commercially supplied with the same dimensions as the PMN-PT plates (Baotou Rare Earth Research Institute, China). It had the highly magnetostrictive [112] crystallographic axis along the length direction so that magnetization (M) was relatively easy in this direction. The piezomagnetic coefficient $\left(d_{33, \mathrm{M}}\right)$, elastic compliance coefficient at constant magnetic field strength $\left(s_{33}^{H}\right)$ and relative magnetic permeability at constant stress $\left(\mu_{33}^{T} / \mu_{0}\right)$ of the Terfenol-D plate were evaluated to be $7.8 \mathrm{~nm} / \mathrm{A}, 27.6 \mathrm{pm}^{2} / \mathrm{N}$ and 4.5 , respectively, at an optimal magnetic bias field of 350 Oe.

In operation, applying an ac voltage $\left(V_{3}\right)$ to the input terminals of the composite produces two equimagnitude, opposite-sign ac electric fields across the thickness of the PMN-PT plates, resulting in transverse piezoelectric strains in the PMN-PT plates owing to the converse piezoelectric effect. These piezoelectrically induced strains lead to mechanical stresses in the sandwiched Terfenol-D plate, giving rise to a magnetic induction $\left(B_{3}\right)$ along the length of the composite because of the converse magnetostrictive effect. To enable description of the composite for magnetic flux/force control applications, one can determine its $\alpha_{\mathrm{B}}$ at frequencies well below its fundamental shape resonance as follows: $:^{12)}$

$$
\alpha_{\mathrm{B}}=\left|\frac{\mathrm{d} B_{3}}{\mathrm{~d} V_{3}}\right|=\left|\frac{d_{31, \mathrm{P}} d_{33, \mathrm{M}}}{s_{11, \mathrm{P}}^{\mathrm{E}} t_{\mathrm{M}}+2 s_{33, \mathrm{M}}^{\mathrm{H}} t_{\mathrm{P}}-2 \frac{d_{33, \mathrm{M}}{ }^{2}}{\mu_{33}^{T}} t_{\mathrm{P}}}\right|
$$

where $t_{\mathrm{M}}$ is the thickness of the Terfenol-D plate, $t_{\mathrm{P}}$ the thickness of the PMN-PT plates, and $\mu_{33}^{T}$ the magnetic permeability at constant stress. Substituting the corresponding material and geometric parameters into Eq. (1), $\alpha_{\mathrm{B}}$ of the proposed composite is predicted to be $0.80 \mathrm{G} / \mathrm{V}$ at $H_{\text {Bias }}=350 \mathrm{Oe}$.

\subsection{Converse magnetoelectric properties}

The CME properties of the composites were characterized using an in-house automated measurement system. ${ }^{13)} \mathrm{A}$ composite sample was placed between the pole gap of a water-cooled, U-shaped electromagnet (Myltem PEM-8005K). By energizing the electromagnet with a dc current supply (Sorensen DHP200$15)$, a magnetic bias field ( $\left.H_{\text {Bias }}\right)$ was generated in the pole gap and was measured with a first Hall probe connected to a Gaussmeter (F. W. Bell 7030). A dynamic signal analyzer (Ono Sokki CF5220) connected to a voltage amplifier (NF-HAS 4025) was employed to provide a $V_{3}$ at the desired amplitude and frequency for the PMN-PT plates of the sample. The corresponding $B_{3}$ induced in the Terfenol-D plate of the sample was measured by
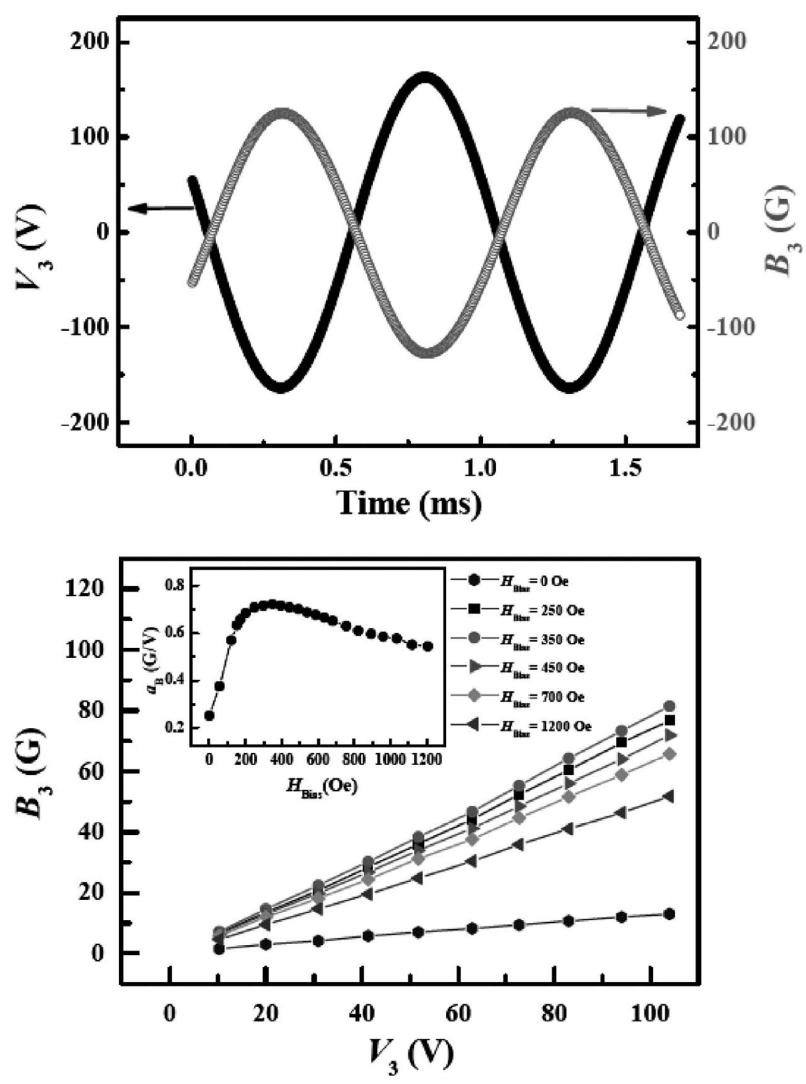

Fig. 2. Measured CME properties of the ME laminated composite in Fig. 1. (a) Waveforms of $B_{3}$ due to an applied $V_{3}$ of $104 \mathrm{~V}$ peak at $1 \mathrm{kHz}$ and with $H_{\text {Bias }}=350$ Oe. (b) $B_{3}$ as a function of $V_{3}$ for various $H_{\text {Bias }}$ at 1 $\mathrm{kHz}$. The inset shows $\alpha_{\mathrm{B}}$ as a function of $H_{\text {Bias. }}$.

a second Hall probe attached to the end of the sample and connected to the dynamic signal analyzer via the Gaussmeter. From the slope of $B_{3}-V_{3}$ plot, $\alpha_{\mathrm{B}}$ was determined. By plotting $\alpha_{\mathrm{B}}$ as a function of $H_{\text {Bias }}$, the $H_{\text {Bias }}$ required to optimally operate the sample was obtained.

Figure 2(a) illustrates the waveforms of the measured $B_{3}$ due to an applied $V_{3}$ of $104 \mathrm{~V}$ peak at $1 \mathrm{kHz}$ and with $H_{\text {Bias }}=350$ Oe. It is clear that $B_{3}$ follows steadily $V_{3}$ and has the maximum amplitude of $81 \mathrm{G}$ when $V_{3}$ peaks at $104 \mathrm{~V}$. The fact that $B_{3}$ and $V_{3}$ are of opposite phase can be explained by the negative sign of the piezoelectric coefficient $d_{31, \mathrm{P}}$ in Eq. (1). Figure 2(b) plots the measured $B_{3}$ as a function of $V_{3}$ for various $H_{\text {Bias }}$ at $1 \mathrm{kHz}$. $B_{3}$ has good linear responses to $V_{3}$ in the entire $V_{3}$ range of 10 $104 \mathrm{~V}$ for all $H_{\text {Bias. }}$. From the slopes of the $B_{3}-V_{3}$ plots, $\alpha_{\mathrm{B}}$ at each $H_{\text {Bias }}$ is determined and shown in the inset of Fig. 2(b). It is seen that $\alpha_{\mathrm{B}}$ increases initially up to $0.73 \mathrm{G} / \mathrm{V}$ at a relatively low $H_{\text {Bias }}$ of $350 \mathrm{Oe}$ and then decreases with increasing $H_{\text {Bias. }}$ The characteristic variation of $\alpha_{\mathrm{B}}$ with $H_{\mathrm{Bias}}$ is mainly attributed to the $H_{\text {Bias- }}{ }^{-}$ dependent piezomagnetic $d_{33, \mathrm{M}}$ contribution from the Terfenol-D plate as a result of the increased and decreased motions of the non- $180^{\circ}$ domain walls. ${ }^{14)}$ Nevertheless, the measured $\alpha_{\mathrm{B}}$ agrees well with the predicted value of $0.80 \mathrm{G} / \mathrm{V}$ at $H_{\text {Bias }}=350$ Oe based on Eq. (1). It is noted that the composite exhibits significantly small variations in $\alpha_{\mathrm{B}}$ (i.e., $0.71-0.73 \mathrm{G} / \mathrm{V}$ ) in the $H_{\text {Bias }}$ range of 250-450 Oe. In order to obtain a high and stable $B_{3}$ in practice, this specific range of $H_{\text {Bias }}$ should be utilized in the design and operation of the composite and its associated devices. 
3. Electromagnetic control device with magnetoelectric laminated composite

3.1 Structure and working principle

Figure 3 shows the solid model and photograph of the proposed electromagnetic control device. The device has a length of $30 \mathrm{~mm}$, a width of $19 \mathrm{~mm}$ and a thickness of $5 \mathrm{~mm}$. A ME laminated composite of PMN-PT and Terfenol-D described in Section 2 is used as the control element and integrated into a magnetic circuit composed of an Alnico biasing magnet (14 $\mathrm{mm} \times 7$ $\mathrm{mm} \times 2 \mathrm{~mm}$ ), two Mn-Zn F-yokes and a Mu-metal movable plate $(30 \mathrm{~mm} \times 5 \mathrm{~mm} \times 0.25 \mathrm{~mm}$ ). The magnetic circuit with the composite in place essentially forms two sets of magnetic flux loops: one including the biasing magnet, the upper section of the Fyokes and the composite (Loop 1), and the other containing the biasing magnet, the lower section (or the output poles) of the Fyokes, the air gaps and the movable plate (Loop 2). The type and size of the biasing magnet have been carefully selected to serve two main purposes: (1) It provides an optimal magnetic bias field $\left(H_{\text {Bias }}\right)$ of 350 Oe to the composite so as to enable a high and stable $\alpha_{\mathrm{B}}$ value $(0.71-0.73 \mathrm{G} / \mathrm{V})$ centering the specific $H_{\text {Bias }}$ range (250-450 Oe) as discussed in Section 2; and (2) it generates a basis value of dc magnetic induction in the gap $\left(B_{\mathrm{go}}\right)$ between the output poles of the F-yokes and the movable plate. For a given gap size $(g)$, the sum of magnetic fluxes in the two loops is

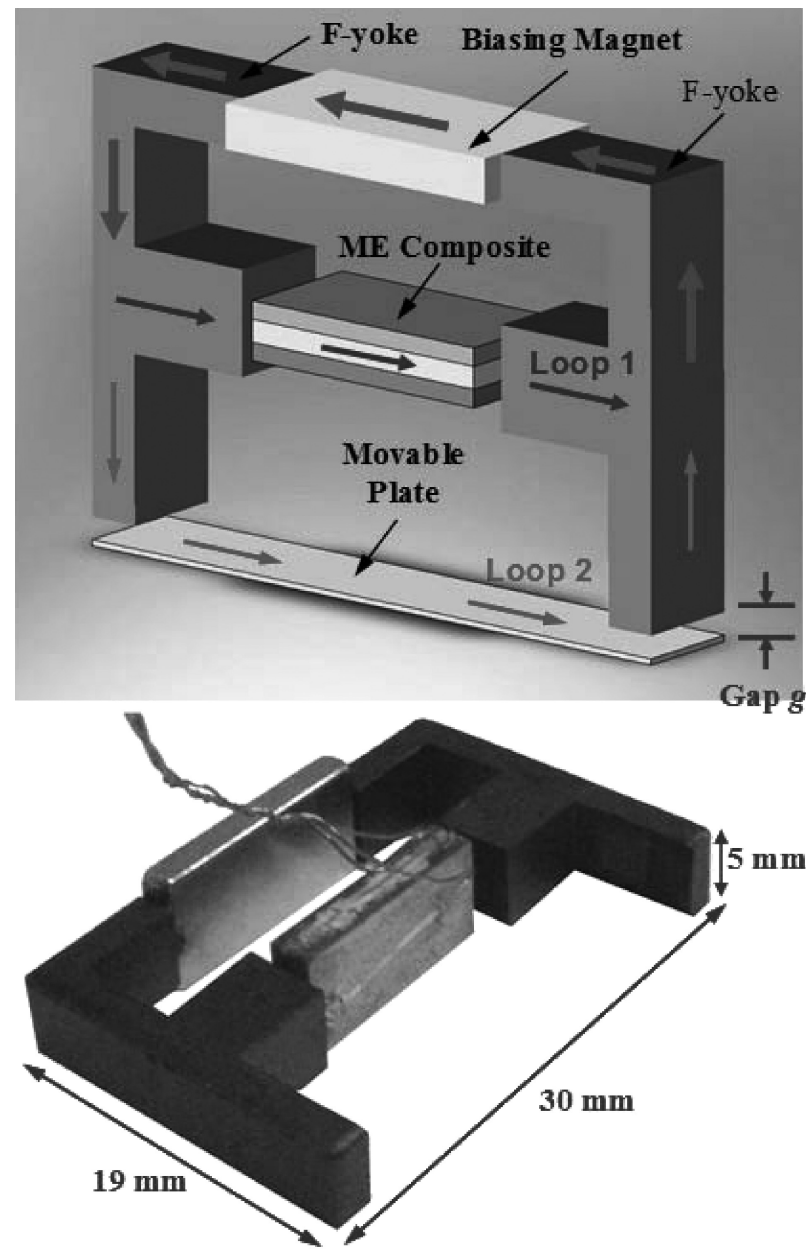

Fig. 3. Solid model (upper) and photograph (lower) of the proposed electromagnetic control device. Two sets of magnetic flux loops (Loop 1 and Loop 2) are created. $g$ is the gap between the output poles of the Fyokes and the movable plate. approximately conserved so that an induced $B_{3}$ in the composite due to an applied $V_{3}$ leads to a change in the magnetic induction in the gap $\left(B_{\mathrm{g}}\right)$ and hence an associated magnetic force acting on the movable plate $\left(F_{\mathrm{g}}\right)$. In applications, this movable plate may be adhered on the surface of the object to be controlled.

\subsection{DC magnetic analysis}

To gain insight into the design of the device with both the composite and magnetic circuit, an ANSYS 10.0 finite element model was created to analyze the distribution of the dc magnetic flux lines of the device, the dc magnetic induction in the gap $\left(B_{\mathrm{go}}\right)$ and the dc magnetic field strength used to bias the composite $\left(H_{\text {Bias }}\right)$, all for different gap sizes $(g)$. The SOLID98 element

Table 1. Magnetic Properties of the Constituent Components of the Proposed Electromagnetic Control Device for the dc Magnetic Finite Element Analysis

\begin{tabular}{cccc}
\hline $\begin{array}{c}\text { Constituent } \\
\text { components }\end{array}$ & Materials & $\begin{array}{c}\text { Relative magnetic } \\
\text { permeability } \mu_{33}^{T} / \mu_{0}\end{array}$ & $\begin{array}{c}\text { Coercive field } \\
H_{C}(\mathrm{Oe})\end{array}$ \\
\hline Magnetoelectric & PMN-PT & 1 & - \\
laminated composite & Terfenol-D & 4.5 & - \\
Biasing magnet & Alnico & 5.3 & 1,670 \\
F-yoke & Mn-Zn ferrite & 2,000 & - \\
Movable plate & Mu-metal & 20,000 & - \\
Air gap & Air & 1 & - \\
\hline
\end{tabular}
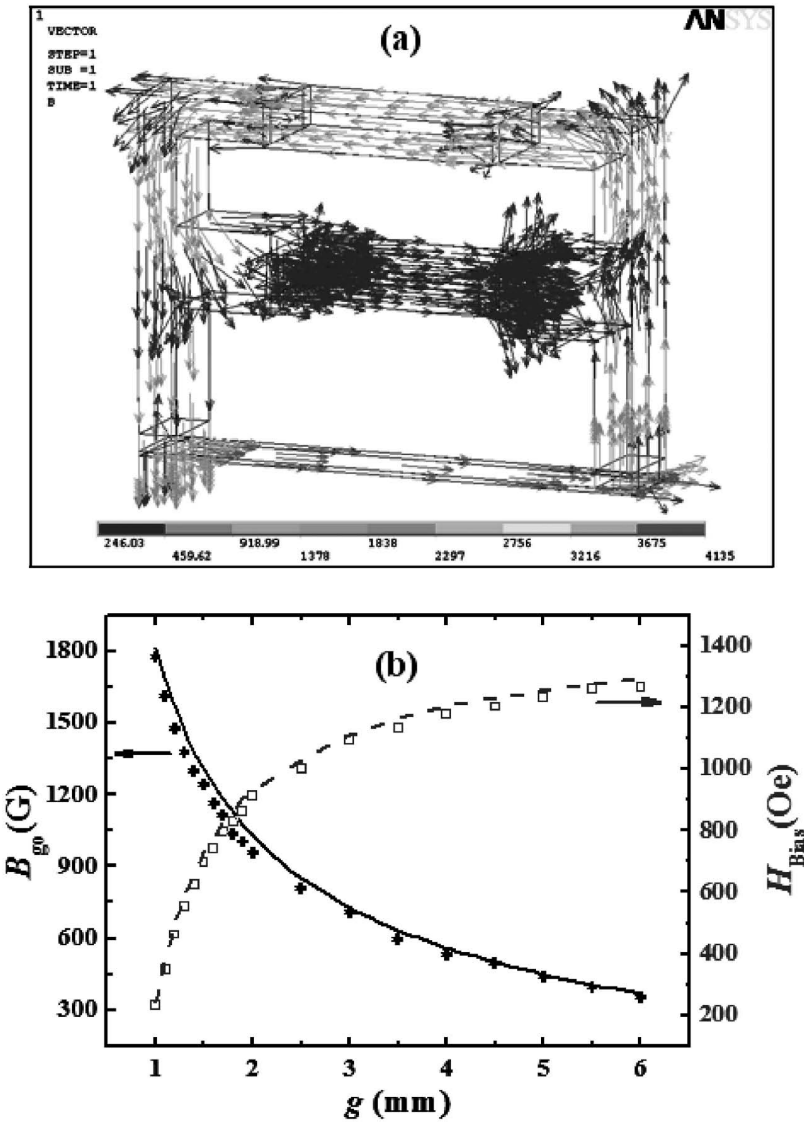

Fig. 4. DC magnetic analysis results. (a) Computed dc magnetic flux lines for a full device model with $g=1.1 \mathrm{~mm}$. (b) Comparison between the computed (lines) and measured (symbols) $B_{\mathrm{go}}$ and $H_{\text {Bias }}$ as a function of $g$. 
was employed for the magnetic analysis. Magnetic properties of the constituent components of the device listed in Table 1 were input into the model.

Figure 4(a) displays an example of the computed dc magnetic flux lines for a full device model with $g=1.1 \mathrm{~mm}$. It is seen that the magnetic circuit incorporated with the composite results in two sets of magnetic flux loops in good agreement with the schematic illustration in Fig. 3. The magnetic circuit is capable of channeling the magnetic fluxes into the composite and the movable plate (via the output poles of the F-yokes). Figure 4(b) plots the comparison between the computed and measured $B_{\mathrm{go}}$ and $H_{\text {Bias }}$ as a function of $g . B_{\text {go }}$ and $H_{\text {Bias }}$ were measured with two separate Hall probes connected to a Gaussmeter (F. W. Bell 7030). This was achieved by placing a first Hall probe on the surface of the movable plate underneath the output pole of an Fyoke and by attaching a second Hall probe to the end of the composite. It is found that the computed $B_{\text {go }}$ and $H_{\text {Bias }}$ coincide well with the measured $B_{\text {go }}$ and $H_{\text {Bias. }}$. There are a gradual decrease in $B_{\text {go }}$ and a corresponding increase in $H_{\text {Bias }}$ with increasing $g$. The complementary trends elucidate the conservation nature of the sum of magnetic fluxes in the two loops. The degree of conservation reduces with increasing $g$ as a result of the increased flux leakage and non-uniformity of flux distributions. Nevertheless, when $g=1.0-1.2 \mathrm{~mm}$ is used, the composite will be biasing in the specific $H_{\text {Bias }}$ range of $250-450$ Oe with a remarkable $\alpha_{\mathrm{B}}$ in excess of $0.7 \mathrm{G} / \mathrm{V}$ (Fig. 2).

\subsection{Performance measurements}

The dynamic performance of the fabricated electromagnetic control device in Fig. 3 was characterized by measuring its ac magnetic induction in the gap $\left(B_{\mathrm{g}}\right)$ and the associated generative force exerted on the movable plate $\left(F_{\mathrm{g}}\right)$ as functions of $V_{3}, g$ and frequency $(f)$. Figure 5 shows the measurement setup. The device was mounted on a $z$-manipulator and positioned right above its movable plate affixed to a load cell (KISTLER Type 9222). $V_{3}$ (and $f$ ) were supplied by a dynamic signal analyzer (Ono Sokki CF5220) via a voltage amplifier (NF-HAS 4025). $B_{\mathrm{g}}$ and $F_{\mathrm{g}}$ were measured using a Hall probe connected to a Gaussmeter (F. W. Bell 7030) and the load cell linked to a signal conditioner (VISHAY 3800), respectively. The acquired $B_{\mathrm{g}}$ and $F_{\mathrm{g}}$ signals were gathered by the dynamic signal analyzer. The exact value of $g$ was confirmed by a laser displacement sensor (Keyence LK-G82) with a controller (Keyence LK-G3001).

Figure 6(a) shows the waveforms of the measured $B_{\mathrm{g}}$ and its excitation $V_{3}$ of $160 \mathrm{~V}$ peak at $1 \mathrm{kHz}$ for $g=1.1 \mathrm{~mm}$. Similar to the observation on the waveforms of $B_{3}$ and $V_{3}$ of the composite

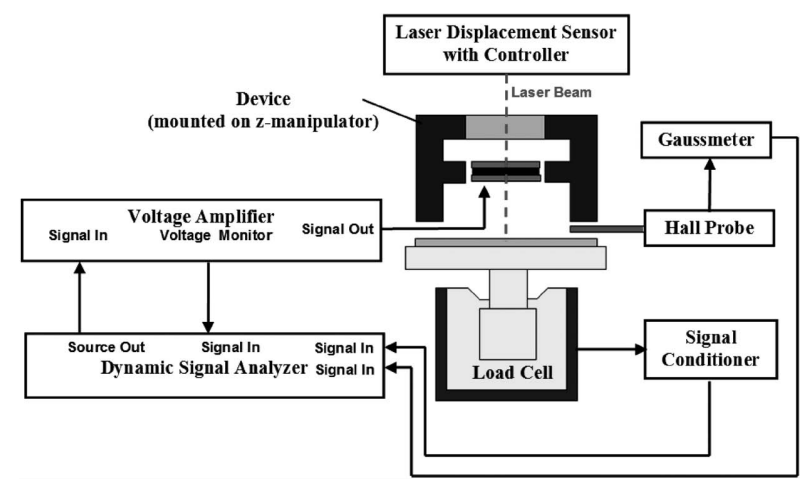

Fig. 5. Measurement setup for characterizing the dynamic performance of the fabricated electromagnetic control device in Fig. 3.
(Fig. 2(a)), $B_{\mathrm{g}}$ and $V_{3}$ of the device, while stable, are of opposite phase with the maximum amplitudes of $104 \mathrm{G}$ and $160 \mathrm{~V}$, respectively. This indicates that an applied positively phased $V_{3}$ produces a negatively phased $B_{3}$ in the composite and hence a negatively phased $B_{\mathrm{g}}$ in the device. Figure 6(b) plots $B_{\mathrm{g}}$ as a function of $V_{3}$ for various $g$ at $1 \mathrm{kHz}$. As expected, $B_{\mathrm{g}}$ has an essentially linear relationship with $V_{3}$ in the measured range of 10-160 V for all $g$. The inset of Fig. 6(b) relates the CME flux coefficient of the device $\left(\alpha_{\mathrm{Bg}}\right)$, defined by a magnetic induction in a given gap in response to an applied ac voltage $\left(\mathrm{d} B_{\mathrm{g}} / \mathrm{d} V_{3}\right)$, with $g$. It is obvious that $\alpha_{\mathrm{Bg}}$ attains its generally high value in excess of 0.6 $\mathrm{G} / \mathrm{V}$ in the $g$ range of $1.0-1.2 \mathrm{~mm}$. This range of $g$ corresponds
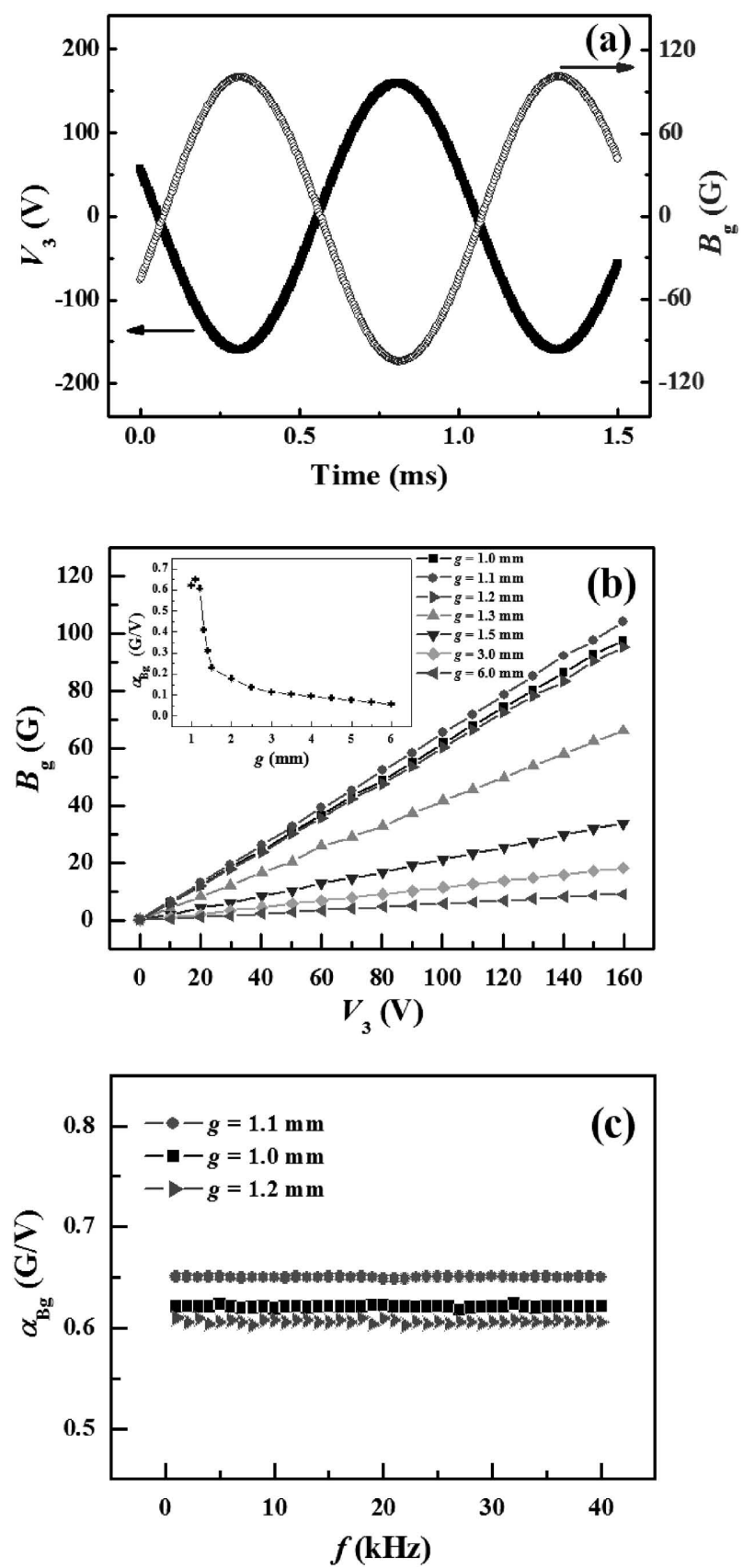

Fig. 6. Measured dynamic magnetic flux results. (a) Waveforms of $B_{g}$ and its excitation $V_{3}$ of $160 \mathrm{~V}$ peak at $1 \mathrm{kHz}$ for $g=1.1 \mathrm{~mm}$. (b) $B_{\mathrm{g}}$ as a function of $V_{3}$ for various $g$ at $1 \mathrm{kHz}$. The inset relates $\alpha_{\mathrm{Bg}}$ with $g$. (c) $f$ dependence of $\alpha_{\mathrm{Bg}}$ at three different $g$ of 1.0,1.1 and $1.2 \mathrm{~mm}$. 


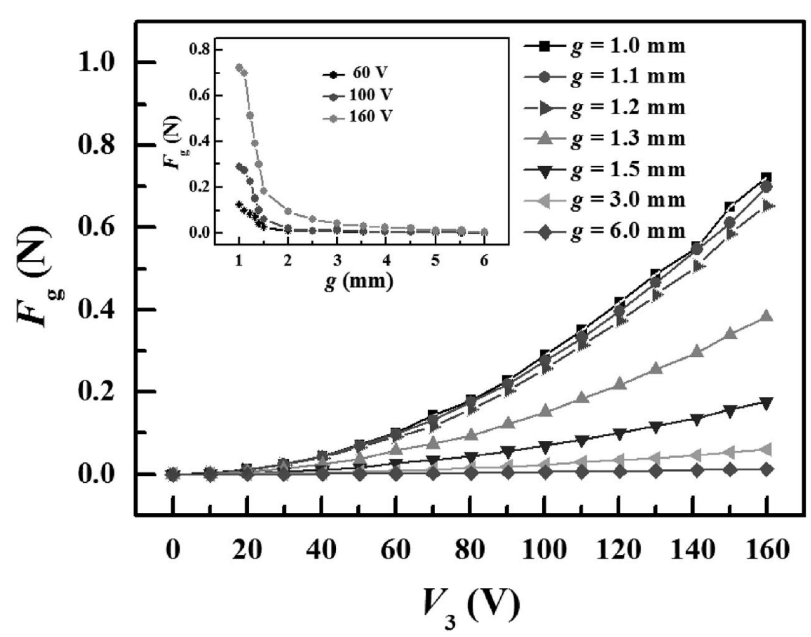

Fig. 7. Measured generative force results: $F_{\mathrm{g}}$ versus $V_{3}$ for various $g$ at $1 \mathrm{kHz}$. The inset shows the variation of $F_{\mathrm{g}}$ with $g$ at three different $V_{3}$ of 60,100 and $160 \mathrm{~V}$

to biasing the composite in the $H_{\text {Bias }}$ range of 250-450 Oe with a generally high and stable $\alpha_{\mathrm{B}}$ of $0.71-0.73 \mathrm{G} / \mathrm{V}$. Figure $6(\mathrm{c})$ illustrates the $f$ dependence of $\alpha_{\mathrm{Bg}}$ for three different $g$ of 1.0, 1.1 and $1.2 \mathrm{~mm}$. It is noted that $\alpha_{B \mathrm{~g}}$ has essentially flat responses in the measured $f$ range of $0.1-40 \mathrm{kHz}$ with no observable dispersion of the CME effect.

Figure 7 shows the relationship between $F_{\mathrm{g}}$ and $V_{3}$ for various $g$ at $1 \mathrm{kHz}$. Instead of exhibiting a highly linear relationship with $V_{3}$ as in Fig. 2(b) for $B_{3}$ and in Fig. 6(b) for $B_{\mathrm{g}}, F_{\mathrm{g}}$ demonstrates nonlinear increasing trends with $V_{3}$. These nonlinear trends may be explained by Eq. (2) in which $F_{\mathrm{g}}$ is a function of both $B_{\mathrm{g}}$ and $B_{\text {go }}$ rather than a function of $B_{\mathrm{g} .}{ }^{10)}$

$$
F_{\mathrm{g}}=\frac{2 B_{\mathrm{g}} B_{\mathrm{go}} A_{\mathrm{g}}}{\mu_{o}}
$$

where $A_{\mathrm{g}}$ is the cross-section area of the output poles of the Fyokes and $\mu_{\mathrm{o}}\left(=4 \pi \times 10^{-7} \mathrm{H} / \mathrm{m}\right)$ the magnetic permeability at free space. From the inset of Fig. 7, it is known that $F_{\mathrm{g}}$ is capable of maintaining stable and high values at the designated gaps of 1.0$1.2 \mathrm{~mm}$.

\section{Conclusions}

We have developed, analyzed and characterized a coil-free electromagnetic control device based on the CME in a ME laminated composite of a Terfenol-D alloy plate sandwiched between two electro-parallel-connected PMN-PT single crystal plates in the thickness direction. The composite demonstrates a high $\alpha_{\mathrm{B}}$ of $0.73 \mathrm{G} / \mathrm{V}$ at a relatively low $H_{\text {Bias }}$ of $350 \mathrm{Oe}$, together with an excellent linear relationship between $B_{3}$ and $V_{3}$ in the measured $V_{3}$ range of $10-104 \mathrm{~V}$ for $H_{\text {Bias }}$ varying from 0 to 1200 Oe. The device shows generally high $\alpha_{\mathrm{Bg}}$ over $0.6 \mathrm{G} / \mathrm{V}$ with flat frequency responses in the measured range of $0.1-40 \mathrm{kHz}$ and with large generative forces in excess of $0.7 \mathrm{~N}$ at $160 \mathrm{~V}$ at the designated gaps of 1.0-1.2 mm. Comparing to conventional coilwound electromagnetic devices based on Faraday's law of electromagnetic induction, our device has the distinct advantage of no magnetic coil, and is thus suitable for magnetic flux/force control applications where reduced Joule heating losses, improved operating bandwidths and increased control preciseness are of great concern/interest.

Acknowledgements This work was supported by the Research Grants Council of the HKSAR Government (PolyU 5122/05E), The Hong Kong Polytechnic University (1-BB95), the 863 High Technology and Development Project of the People's Republic of China (2006AA03Z107), the Natural Science Foundation of China (50432030, 50777065 and 50602047), the Scientific Innovation Program of Chinese Academy of Sciences (KGCX2-YW-111-7), Shanghai Municipal Government (06DZ05016), and Innovation Funds from Shanghai Institute of Ceramics of Chinese Academy of Sciences (SCX0608).

\section{References}

1) L. D. Landau and E. Lifshitz, "Electrodynamics of Continuous Media," Pergamon, Oxford (1960).

2) V. J. Folen, G. T. Rado and E. W. Stalder, Phys. Rev. Lett., 6, 607 (1961).

3) J. Ryu, A. V. Carazo, K. Uchino and H. Kim, J. Electroceram., 7, 24 (2001).

4) S. Dong, J. F. Li and D. Viehland, Appl. Phys. Lett., 83, 2265 (2003).

5) S. W. Or and N. Cai, Solid State Phenom., 111, 147 (2006).

6) T. Li, S. W. Or and H. L. W. Chan, J. Magn. Magn. Mater., 304, e442 (2006).

7) S. W. Or and H. L. W. Chan-Wong, US Patent, US7, 199, 495B2 (2007).

8) Y. M. Jia, S. W. Or, H. L. W. Chan, X. Y. Zhao and H. S. Luo, Appl. Phys. Lett., 88, 242902 (2006).

9) W. Y. Wong, S. W. Or, H. L. W. Chan, Y. M. Jia and H. S. Luo, J. Appl. Phys., 101, 09N508 (2007).

10) E. P. Furlani, "Permanent Magnet and Electromechanical Devices: Materials, Analysis, and Applications," Academic, San Diego (2001).

11) J. Peng, H. S. Luo, D. Lin, H. Q. Xu, T. H. He and W. Q. Jin, Appl. Phys. Lett., 85, 6221 (2004).

12) W. Y. Wong, "MPhil Thesis," The Hong Kong Polytechnic University (2007).

13) S. W. Or, T. L. Li, and H. L. W. Chan, J. Appl. Lett., 97, 10M308 (2005).

14) S. W. Or and G. P. Carman, IEEE Trans Magn., 41, 27902792 (2005). 